\title{
Positron lifetime spectroscopy applied to pure Tellurium
}

\author{
R Domínguez-Reyes* ${ }^{\mathbb{D}}$ \\ Departamento de Física, Universidad Carlos III de Madrid, Avda. de la Universidad 30, 28911 Leganés, Madrid, Spain
}

Received: 18 March 2021 / Accepted: 22 July 2021 / Published online: 15 August 2021

\begin{abstract}
High-purity crystalline Tellurium has been investigated using positron lifetime spectroscopy technique in order to determine basic information missing in the current experimental knowledge of the positron annihilation spectroscopy field. Three different pairs of samples have been studied in the as-received state and, in order to eliminate the vacancy-type defects, after consecutive isothermal treatments at $300{ }^{\circ} \mathrm{C}$. Lifetime corresponding to the annihilation in the Tellurium bulk has been determined as 282(1) ps. Previous theoretical calculations present in the bibliography that used different methods and parameterization provided a wide range of values for the annihilation lifetime of the positron in the bulk of Tellurium. The obtained result has been used to identify the most accurate results among them.
\end{abstract}

Keywords: Positron annihilation spectroscopy; Positron lifetime; Tellurium; Bulk

\section{Introduction}

Tellurium (Te) is Group VI metalloid or semiconductor with piezoelectric [1], piezo-resistance [2], and photoconductivity [3] properties. This makes Te suitable for many different uses in material sciences due to its unique properties. As a major element it can be used in the form of Tellurites for laser generation [4] or in Tellurides for solar cell applications $[5,6]$. It is also used as a minor element doping different semiconductors such as GaSb [7] or InGaAs [8] in order to improve the photovoltaic properties.

Positron annihilation spectroscopy (PAS) is an advanced characterization technique widely used in metals and metallic alloys [9], semiconductors [10], polymers [11], or dielectrics [12], and in many applications such as solar cells [13], photonics [14], or electronic materials [15] among others, PAS allows to obtain information about the lattice defects (from single vacancies to vacancy clusters) as they act as traps for thermalized positrons. Positron lifetime spectroscopy (PLS) provides information about the size and abundance of these types of defects providing longer lifetime components as the size of the defect grows. Nevertheless, positrons can also annihilate in the bulk of the materials if the defects density is not high enough to saturate the positron trapping, resulting in lifetime components shorter than defects' lifetimes.

*Corresponding author, E-mail: rdomingu@ fis.uc3m.es
PAS has been applied in many studies to complex materials that included Te [16, 17], but even when PLS theoretical calculations exist [18], some basic experimental knowledge about pure Te studied by PAS, such as the lifetime associated with the annihilation in the bulk of the material, is still absent. The aim of this experimental work is determining the lifetime associated with the annihilation in the bulk of pure Te as a continuation of a previous work where the coincidence Doppler broadening (CDB) signature curve was recently obtained [19].

\section{Experimental details}

A $0.5 \mathrm{MBq} 22 \mathrm{Na}$ source sealed in kapton was placed between couples of samples of $99.99 \%$ purity crystalline Te. Three couples of Te samples were prepared (at least $10 \mathrm{~mm} \times 10 \mathrm{~mm}, 5 \mathrm{~mm}$ thick) and measured independently to improve the reliability of the study. The samplessource system was placed in the centre of a fast-fast coincidence spectrometer used in previous studies [20,21], consisting of two plastic scintillators face-to-face. Fe reference samples (99.999\% purity annealed during $3 \mathrm{~h}$ at 800 ${ }^{\circ} \mathrm{C}$ ) were used to test the system before and after the $\mathrm{Te}$ samples measurements, resulting in a lifetime value of $118(1)$ ps (slightly higher than the perfect Fe lattice lifetime around $110 \mathrm{ps}[18,22])$. The Fe reference sample was also used to determine the time resolution ( $240 \mathrm{ps})$. Mo reference sample $(99.999 \%$ purity annealed during $3 \mathrm{~h}$ at 
$1400{ }^{\circ} \mathrm{C}, 119(1)$ ps lifetime against 120 ps reference value [23]) was used to determine the source correction contribution to the lifetime components, that is required to analyse any PLS spectra, resulting on 382(1) ps with intensity 17.9(1)\%. Mo was used instead of Fe for a better determination of the source correction as its atomic number $(Z=42)$ is closer to Te atomic number $(Z=52)$ and it influences the positron reflection in the surface of the material [24, 25]. Additionally, low-intensity long-lifetime component (around 2400 ps) associated with source sealing material and surface effect was also determined as an extra component associated with the source. Cumulative spectra (without electronic drift during or between the measurements) with above $1 \times 10^{6}$ counts were obtained for each pair of samples. The resulting data were analysed using PASFIT-88 [26] considering the possible existence of several contributions to the spectrum and choosing the best possible fit (Chi-square normalized to the number of data and degrees of freedom better than $5 \times 10^{-3}$ ). The obtained data statistics allowed the identification of lowintensity lifetime components down to $2 \%$ contribution.

\section{Results and discussion}

\subsection{As-received state samples}

The pairs of samples, prepared and measured independently to improve the consistency of the conclusions, resulted in analogous results for all of them. These results can be found in Table 1. As the results are homogeneous, their average (also present in Table 1) was used as a unique value in the discussion for simplicity. Statistical dispersion between sample's individual results was considered. Error provided by the fit resulted in being equal or higher than the statistical dispersion, so it has been considered as the main error source for the measurements and used as the error bar for the average values.

Best fit of the spectra (example of the fit can be seen in Fig. 1(a)) resulted in two components ( $\tau_{1}$ and $\tau_{2}$ from now) after considering the two components associated with the source. Considering one or more than two components

Table 1 Fitted lifetime parameters for the three pairs Te samples in as-received state

\begin{tabular}{lllll}
\hline Sample & $\tau_{1}(\mathrm{ps})$ & $I_{1}(\%)$ & $\tau_{2}(\mathrm{ps})$ & $I_{2}(\%)$ \\
\hline Te (as-received) & $278(2)$ & $81(9)$ & $580(80)$ & $19(9)$ \\
& $281(2)$ & $81(9)$ & $590(80)$ & $19(9)$ \\
& $276(2)$ & $82(9)$ & $590(90)$ & $18(9)$ \\
Average & $278(2)$ & $81(10)$ & $590(90)$ & $19(10)$ \\
\hline
\end{tabular}

associated with the sample resulted in non-reliable fits. Long-life component $\tau_{2}$, with average value 590(90) ps, is significantly higher than the reported lifetime for single vacancy 339 ps [27, 28].

Due to its high value, this lifetime component should also be associated with positronium. Positronium [29] is a non-stable hydrogen-like bound state between a positron and an electron. There are two possible spin states for positronium, a singlet spin state, called para-positronium; and a triplet spin state, called ortho-positronium. Positronium is formed in regions with low electronic density, such as pores or big vacancy clusters that can be typically found in materials such as Te. Lifetime associated with parapositronium in void (125 ns) is significantly lower than the lifetime associated with ortho-positronium (142 ns). Nevertheless, these lifetimes refer to positronium in void, and they can be reduced by the presence of an atomic environment by the pick-off annihilation that can reduce the ortho-positronium lifetime to nearly 600 ps [30] in some cases (any case above 500 ps). Second lifetime components (580-610 ps) seem to correspond to ortho-positronium. Shorter lifetime components associated with parapositronium were not detected, probably because of its low intensity.

The short-lifetime component $\tau_{1}$, with value average 278(2) ps, is significantly lower than the already mentioned single-vacancy lifetime (339 ps [27, 28]). Passivation of single vacancies by the presence of hydrogen $(\mathrm{H})$ filling them reduces the observed lifetime in semiconductors and other materials [31-33]. Even when this could explain this short-lifetime component, molecular $\mathrm{H}$ doesn't adsorb or react with $\mathrm{Te}$ in the studied temperature range [34], so some other possibility should be considered as no evidence of the presence of $\mathrm{H}$ seems to be reasonable considering the preparation and treatment of Te samples. An alternative assignment to this short-lifetime component could be the annihilation in the bulk as no other annihilation place should have such a lower lifetime (as annihilation on dislocations has a lifetime similar to annihilation on singlevacancy). Initial PAS work in semiconductors provided an experimental value for Te positron lifetime of 358(10) ps [35]. This was tentatively associated with annihilation in bulk, but considering the later reported single-vacancy value (339 ps [27, 28]), and the fact the analysed Te was powder (instead of crystalline Te used in this work), it is probably a mis-assignation as bulk lifetime cannot be higher than single-vacancy lifetime.

Even when this component could be associated with the annihilation in the Te bulk, in the possible presence of defects (as indicated by the presence of the positronium component) its value cannot be directly assigned to it because of the influence of the positron trapping rate in the exact type of defects present in the samples [36]. 

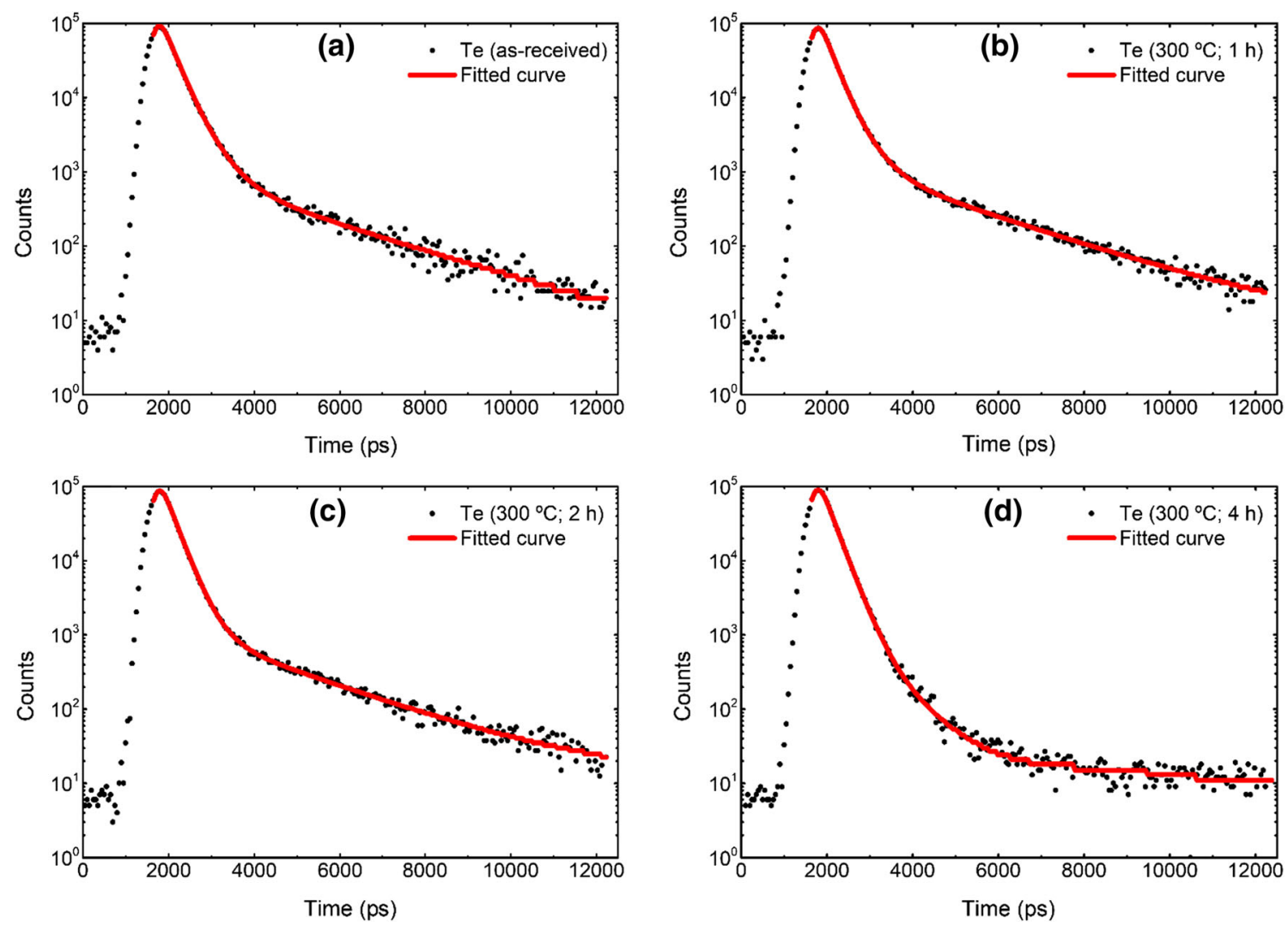

Fig. 1 PLS data and fitted curve for selected samples: (a) as-received state, (b) after 1-h annealing, (c) after 2-h annealing and (d) after 4-h annealing (evidencing the decrease of the lifetime by the absence of the $\tau_{2}$ component)

\subsection{Thermal treated samples}

Based on the previous result, and in order to reduce the possible defects contribution, the samples were measured

Table 2 Fitted lifetime parameters for the three pairs Te samples annealed at $300{ }^{\circ} \mathrm{C}$ for $1 \mathrm{~h}, 2 \mathrm{~h}$, and $4 \mathrm{~h}$

\begin{tabular}{lllll}
\hline Sample & $\tau_{1}(\mathrm{ps})$ & $\mathrm{I}_{1}(\%)$ & $\tau_{2}(\mathrm{ps})$ & $I_{2}(\%)$ \\
\hline Te $\left(300{ }^{\circ} \mathrm{C} ; 1 \mathrm{~h}\right)$ & $275(2)$ & $92(6)$ & $600(100)$ & $8(6)$ \\
& $275(2)$ & $90(5)$ & $590(80)$ & $10(5)$ \\
& $277(2)$ & $92(5)$ & $590(80)$ & $8(5)$ \\
Average & $276(2)$ & $91(6)$ & $600(100)$ & $9(6)$ \\
Te $\left(300{ }^{\circ} \mathrm{C} ; 2 \mathrm{~h}\right)$ & $276(2)$ & $97(2)$ & $610(80)$ & $3(2)$ \\
& $277(1)$ & $97(2)$ & $600(80)$ & $3(2)$ \\
& $277(2)$ & $97(2)$ & $610(90)$ & $3(2)$ \\
Average & $277(2)$ & $97(2)$ & $610(80)$ & $3(2)$ \\
Te $\left(300{ }^{\circ} \mathrm{C} ; 4 \mathrm{~h}\right)$ & $282(1)$ & $100(-)$ & - & - \\
& $282(1)$ & $100(-)$ & - & - \\
Average & $281(1)$ & $100(-)$ & - & - \\
\hline
\end{tabular}

again after isothermal treatment (annealing) in vacuum better than $1.5 \cdot 10-5 \mathrm{mBar}$. As in the previous case, the results (present in Table 2) were homogeneous in each case, so their average will be used for the discussion. Vaporization can be an issue that completely vaporizes the samples at this pressure and temperature range [37], but only a light superficial vaporization effect was observed in the samples. Annealing for longer times or higher temperatures resulted in the destruction of the test samples.

After the first annealing $\left(300^{\circ} \mathrm{C}\right.$ for $\left.1 \mathrm{~h}\right)$, the intensity of the long-life component $\tau_{2}$ associated with positronium decreased from the initial 19(10)-9(6)\% keeping the same lifetime value within the error bar. This decrease in the component seemed to indicate the density of defects was reduced by the thermal treatment, implying that successive annealing could completely anneal out the defects. Further annealing at the same temperature for 1 extra hour $(2 \mathrm{~h}$ total) decreased the intensity of $\tau_{2}$ to $3(2) \%$ keeping again the lifetime value of the component. This long-lifetime component disappeared after 2 extra hours (4 total hours) annealing. As the long-life component associated with positronium progressively annealed out as the cumulative time of the annealing process increased, the association of 
Table 3 Te bulk lifetime calculation for different methods [18]

\begin{tabular}{llll}
\hline Method & Parameterization & Bulk lifetime $(\mathrm{ps})$ & Vacancy lifetime $(\mathrm{ps})$ \\
\hline AT-SUP & BN & 289 & 329 \\
AT-SUP & GGA & 345 & 353 \\
AT-SUP & LDA & 250 & 289 \\
LMTO-ASA & BN & 286 & 350 \\
LMTO-ASA & GGA & 307 & 400 \\
\hline
\end{tabular}

this component to open-volume defects seemed to be confirmed. Examples of the data and fitted curves can be seen in Fig. 1(b), (c), and (d).

During the annealing process, short-lifetime component $\tau_{1}$, remained in the same lifetime values within the error bar until the last annealing where the lifetime increased slightly. If this component was associated with H-decorated single vacancies, it should have been strongly affected by the thermal treatments, as it is unlikely that open-volume defects are completely annealed out and single-vacancies are unaltered (and therefore the lifetime associated with them). This seems to support the assignment of the short-lifetime component to the annihilation in the bulk of the samples. As previously commented, capture rate in defects affects shorter lifetime components; therefore, as defects seem to have been annealed out after the last thermal treatment, the short-lifetime component is slightly affected. This component, with value $282(1) \mathrm{ps}$, is significantly lower than the single-vacancy lifetime (339 ps), any other structures such as dislocations should have annealed out before the vacancy clusters, so it might be safe assigning this value of the short lifetime $\tau_{1}$, as unique lifetime component, to the annihilation in the bulk in the absence of defects that could influence the lifetime by their capture rate.

\subsection{Comparison with theoretical calculation}

The obtained result for the positron annihilation in the Te bulk can be compared to theoretical calculations present in the bibliography. In initial PLS calculations works [38-42], Tellurium was not included, but later works based on them [18] include it among many other elements. Table 3 contains the calculated values for Te bulk and single-vacancy lifetimes for the different models and parameterizations used in [18]. Different methods and parameterizations provided values in a wide range for the bulk lifetime, between 250 and 345 ps.

Atomic superposition approximation (AT-SUP) and linear muffin-tin orbital method within the atomic-spheres approximation (LMTO-ASA) methods [38] with the Boroński and Nieminen correlation energy the interpolation formula $(\mathrm{BN})$ [39] are the best match with the obtained experimental results, $289 \mathrm{ps}$ and $286 \mathrm{ps,}$ respectively. Detailed explanations about calculation can be found in [18] and its base in [38-42]. This value is slightly higher than the value obtained in this work (282(1) ps), but significantly close considering the amplitude range of the variation of the values between methods and parameterizations (95 ps). It should be noted that the mono-vacancy lifetime provided by these models with the BN approximation ( 329 ps and $350 \mathrm{ps)}$ is also a good approximation to the reported experimental value (339 ps [27, 28]).

\section{Conclusions}

High-purity crystalline Te has been investigated by PLS spectroscopy and the annihilation lifetime in the bulk has been determined as 282(1) ps after eliminating defects contribution by successive annealing of the samples. The obtained value, 282(1) ps, has been compared with previous theoretical calculation present in bibliography [6] matching the AT-SUP and LMTO-ASA methods [18, 38] with $\mathrm{BN}$ parameterization [39] that already provided a result for the Te single-vacancy lifetime similar to the experimental value. This new obtained value provides information that can be used as reference in further studies involving Te-based materials.

Acknowledgements This research was supported by the Universidad Carlos III de Madrid.

Funding This research was supported by the Universidad Carlos III de Madrid and by the Comunidad de Madrid (Spain)—-multiannual agreement with UC3M ("Excelencia para el Profesorado Universitario"-EPUC3M14)—Fifth regional research plan 2016-2020.

Data availability The raw/processed data required to reproduce these findings cannot be shared at this time as the data also form part of an ongoing study.

Code availability Not applicable.

\section{Declarations}

Conflict of interest The authors declare that they have no known competing financial interests or personal relationships that could have appeared to influence the work reported in this paper. 
Consent to participate Not applicable.

Consent for publication Not applicable.

Ethics approval Not applicable.

Open Access This article is licensed under a Creative Commons Attribution 4.0 International License, which permits use, sharing, adaptation, distribution and reproduction in any medium or format, as long as you give appropriate credit to the original author(s) and the source, provide a link to the Creative Commons licence, and indicate if changes were made. The images or other third party material in this article are included in the article's Creative Commons licence, unless indicated otherwise in a credit line to the material. If material is not included in the article's Creative Commons licence and your intended use is not permitted by statutory regulation or exceeds the permitted use, you will need to obtain permission directly from the copyright holder. To view a copy of this licence, visit http://creativecommons. org/licenses/by/4.0/.

\section{References}

[1] G Arlt and P Quadflieg Phys. Status Solidi B 32687 (1969)

[2] T Takagi Phys. Status Solidi B 72675 (1975)

[3] D F Edwards, C D Butter and L D McGlauchlin Solid State Electron. 324 (1961)

[4] K Pach, E Golis, E S Yousef, M Sitarz and J Filipecki J. Mol. Struct. 1164328 (2018)

[5] M R M Elsharkawy, G S Kanda, E E Abdel-Hady and D J Keeble Appl. Phys. Lett. 108242102 (2016)

[6] H Li, K Zhou, J Pang, Y Shao, Z Wang and Y Zhao Semicond. Sci. Tech. 26075016 (2011)

[7] L Šedivý, J Č́žek, E Belas, R Grill and O Melikhova Sci. Rep. 6 20641 (2016)

[8] T Orzali et al J. Cryst. Growth 426243 (2015)

[9] R W Siegel Ann. Rev. Mater. Res. 10393 (1980)

[10] R Krause-Rehberg, R K R H S Leipner and H S Leipner Springer Series in Solid-State Sciences, vol 127. (Berlin: Springer) (1999)

[11] Y C Jean Microchem. J. 4272 (1990)

[12] A G Attallah et al Micropor. Mesopor. Mat. 308110457 (2020)

[13] C J Edwardson, P G Coleman, T-TA Li, A Cuevas and S Ruffell J. Appl. Phys. 111053515 (2012)

[14] R Reisfeld, T Saraidarov and B Jasinska Opt. Mater. 26181 (2004)

[15] M Haseman et al J. Electron. Mater. 456337 (2016)

[16] N M Abdul-Jabbar, E D Bourret-Courchesne and B D Wirth $J$. Cryst. Growth 35231 (2012)

[17] K Tang et al J. Appl. Phys. 117135304 (2015)
[18] J M Campillo Robles, E Ogando and F Plazaola J. Phys. Condens. Matter 19176222 (2007)

[19] R Domínguez-Reyes Results Phys. 121455 (2019)

[20] R Domínguez-Reyes, M A Auger, M A Monge and R Pareja Phil. Mag. 97833 (2017)

[21] R Rementeria, R Domínguez-Reyes, C Capdevila, C GarciaMateo and F G Caballero Sci. Rep. 10487 (2020)

[22] A Vehanen, P Hautojärvi, J Johansson and J Yli-Kauppila Phys. Rev. B 25762 (1982)

[23] B Pagh, H E Hansen, B Nielsen, G Trumpy and K Petersen Appl. Phys. A Mater. Sci. Proc. 33225 (1984)

[24] S McGuire and D J Keeble J. Phys. D: Appl. Phys. 393388 (2006)

[25] S McGuire and D J Keeble J. Appl. Phys. 100103504 (2006)

[26] P Kirkegaard, J V Olsen, M M Eldrup and N J Pedersen PALSFIT: A Computer Program for Analyzing Positron Lifetime Spectra (Denmark: Danmarks Tekniske Universitet, Ris $\emptyset$ Nationallaboratoriet for Bæredygtig Energi) (2009)

[27] B K Meyer and W K Stadle J. Cryst. Growth 161119 (1996)

[28] D Verstraeten et al Semiconductor Science and Technology 18 919 (2003)

[29] O E Mogensen Positron Annihilation in Chemistry. (Berlin: Springer) (1995)

[30] T Goworek, B Jasińska, J Wawryszczuk, R Zaleski and T Suzuki Chem. Phys. 280295 (2002)

[31] M M Islam et al Sci. Rep. 106134 (2020)

[32] F A Selim, C R Varney, M C Tarun, M C Rowe, G S Collins and M D McCluskey Phys. Rev. B 88174102 (2013)

[33] R S Brusa et al Phys. Rev. B 497271 (1994)

[34] D A Outka Surf. Sci. 235 L311 (1990)

[35] R Fieschi, A Gainotti, C Ghezzi and M Manfredi Phys. Rev. 175 383 (1968)

[36] M Eldrup J. Phys. IV Colloque 0593 (1995)

[37] R Viswanathan, R Balasubramanian, D Darwin Albert Raj, M Sai Baba and T S Lakshmi Narasimhan J. Alloy. Comp. 60375 (2014)

[38] M J Puska J. Phys. Condens. Matter. 33455 (1991)

[39] E Boroński and R M Nieminen Phys. Rev. B 343820 (1986)

[40] M J Puska and R M Nieminen J. Phys. F Met. Phys. 13333 (1983)

[41] O K Andersen, O Jepsen and D Glötzel Highlight of CondensedMatter Theory (eds.) F Bassani, F Fumi and M P Tosi (New York: North-Holland) (1985)

[42] O K Andersen, O Jepsen and M Sob Electronic Band Structure and Its Applications (ed.) M Yossouff (Heildeberg: Springer) p 1 (1987)

Publisher's Note Springer Nature remains neutral with regard to jurisdictional claims in published maps and institutional affiliations. 